\title{
El periodismo ambiental como área de especialización en las aulas universitarias
}

\author{
Ma Teresa Mercado SÁEz \\ Universidad CEU-Cardenal Herrera \\ mmercado@uch.ceu.es \\ Sebastián SÁNChez CASTILlo \\ Universitat de València \\ Sebastian.sanchez@uv.es \\ José María Herranz De la CaSA \\ Universidad de Castilla-La Mancha \\ josemaria.herranz@uclm.es
}

\begin{abstract}
Resumen:
Informar sobre los asuntos relacionados con el medio ambiente se ha convertido en los últimos años en un área de especialización periodística consolidada al mismo tiempo que la actualidad demuestra que los asuntos ambientales son asuntos políticos, económicos y sociales. La enseñanza universitaria del Periodismo no puede ser ajena a la necesidad de conocer esta realidad transversal. Las exigencias de la implantación del Espacio Europeo de Educación Superior en nuestras universidades obliga a considerar nuevas fórmulas para la integración del medio ambiente en las aulas.
\end{abstract}

Palabras clave: periodismo ambiental; especialización; enseñanza universitaria

\section{Environmental journalism as area of specialization in university classrooms}

\begin{abstract}
:
Report environment issues has become in recent years an consolidated area of specialization in journalism when environment is a political, economic and social question. Journalism education can not ignore the need to know this cross curricular subject. The demands of the European Space of Higher Education in our universities requires consideration of new approaches for integrating environment in the classroom.
\end{abstract}

Key Words: environmental journalism; specialization; university education

\section{Referencia normalizada:}

Mercado Sáez, M. T., Sánchez Castillo, S. y Herránz de la Casa, J. M. (2014): El periodismo ambiental como área de especialización en las aulas universitarias. Historia y Comunicación Social. Vol. 19. Núm. Especial Marzo. Págs. 213-226.

Sumario: 1. Introducción. 2. El Periodismo ambiental como asignatura. 2.1 Los temas del periodismo ambiental: la cuestión energética. 3. La información sobre medio ambiente en los Fundamentos del Periodismo. 3.1. Metodología. 3.2 Resultados. 4. Conclusiones. 5. Bibliografía. 


\section{Introducción}

El periodismo ambiental ha sido un área de especialización poco estudiada desde el punto de vista académico. Nada extraño si consideramos que el propio concepto 'medio ambiente' es relativamente reciente puesto que apareció en la obra del biólogo y filósofo alemán Jakob J. von Uexküll en su libro Umwelt und Innenwelt der Tiere (Medio ambiente y mundo interno de los animales; de um, que significa alrededor y welt, mundo), publicado en 1909. Así, el medio ambiente engloba el conjunto de circunstancias exteriores a un ser vivo, tal como recogía el Diccionario de la Lengua Española, que en su $22^{\mathrm{a}}$ edición (2001) incluye como primera acepción el conjunto de circunstancias culturales, económicas y sociales en que vive una persona.

Los temas que incluye la especialidad son por tanto muy variados y dependen del punto de vista con el que se entienda el medio ambiente. Mientras Francisco Esteve y Javier Fernández del Moral (1998: 296) definían el área como aquella 'sección periodística que se ocupa de todas aquellas noticias relacionadas con la Naturaleza y su defensa", Rogelio Fernández (2003:150) prefiere hablar del "ejercicio o periodismo especializado que atiende la información generada por la interacción del hombre o de los seres vivos con su entorno o del entorno en sí".

La principal aportación teórica al periodismo ambiental como disciplina es precisamente la realizada por Rogelio Fernández Reyes en diversas publicaciones. En su "Reflexión en torno al debate sobre la definición de periodismo ambiental" constataba cómo esta especialización quedaba fuera de la mayor parte de planes de estudio al entenderse "incorporado" en Periodismo Científico (Fernández Reyes, 2003:145).

La Universidad CEU Cardenal Herrera había sido la única en integrar esta especialización en su oferta de asignaturas optativas en el nuevo plan de estudios que echaba a andar en el curso 2000/01. Estas materias cuatrimestrales de seis créditos comenzaron por tanto a impartirse en el curso 2004/05. Junto a Periodismo Político, Cultural, Social o Jurídico, aparecía Periodismo Ambiental.

\section{El Periodismo ambiental como asignatura}

La asignatura Periodismo ambiental apareció en el Plan de Estudios de la licenciatura de Periodismo que se implantó en la Universidad CEU Cardenal Herrera en el curso 2000-01. Después de ser impartida durante diez cursos, se encuentra en proceso de extinción tras la conversión de la licenciatura en Grado en el proceso de Bolonia.

En los entonces denominados objetivos de conocimientos de la materia (curso 2012-13) figuraban conocer los temas que son objeto de cobertura en esta especialidad y sus implicaciones sociales, en especial todo lo relacionado con el Cambio Climático; reflexionar sobre las funciones y disfunciones de la información sobre medio ambiente y su repercusión; comprender las rutinas especializadas y, en particular, el acceso a las fuentes y el tratamiento de la información sobre medioambiente 
en los distintos soportes; analizar los contextos de producción de la información sobre medio ambiente y delimitar los factores de riesgo, especialmente en situaciones de crisis.

Los objetivos de aplicación se referían a la adquisición de competencias en el manejo de fuentes y recursos de divulgación para producir piezas informativas en el área de medio ambiente en distintos soportes: noticias para prensa y revistas especializadas, y reportajes en televisión. También se señalaba la necesidad de escapar del catastrofismo en el tratamiento de este tipo de información.

El temario de los primeros años seguía el esquema básico de las optativas de Quinto Curso que suponían una inmersión en las áreas de especialización periodísticas después de que en Cuarto se impartiera la asignatura troncal de carácter obligatorio y 12 créditos Periodismo Especializado. Así, los contenidos que se impartían eran los que siguen:

Tema 1. Conceptualización

1.1. Los temas de la información ambiental.

1.2. Origen y evolución de la especialidad.

1.3. Funciones y disfunciones de la información medioambiental.

Tema 2. Actores y fuentes en el periodismo ambiental

2.1. Organizaciones no gubernamentales.

2.2. La administración pública y los organismos oficiales.

2.3. El mundo científico

2.3. Empresas, partidos políticos y sindicatos.

2.4. Colectivos y asociaciones.

Tema 3. Formatos y medios para la información sobre medioambiente

3.1. La información ambiental en la prensa diaria

3.2. Las revistas especializadas y los suplementos.

3.3. La especialidad radiofónica.

3.4. El formato audiovisual

3.5. Periodismo ambiental en Internet.

Tema 4. El valor de servicio en el periodismo ambiental.

4.1. La información meteorológica.

4.2. La información turística. Ecoturismo. Periodismo de viajes.

4.3. Divulgación y protección de la naturaleza. 
Tema 5. Informar sobre medioambiente en tiempo de crisis.

5.1. La gestión de la información en tiempo de crisis.

5.2. La catástrofe medioambiental.

5.3. El conflicto ecológico en proyectos con impacto en el medio ambiente.

El primer tema repasaba la historia del Periodismo Ambiental en España (Varillas, 2007) teniendo en cuenta la poca tradición de la especialización, tanto a nivel teórico como profesional, en comparación con otras como Político, Económico o Cultural. Muy importante era el análisis de las funciones sociales del periodismo ambiental: denuncia de las agresiones ocasionadas contra el planeta; divulgación de los descubrimientos técnicos y científicos que favorezcan una mejor protección medioambiental; concienciación ecológica en la sociedad a favor de una conservación (Fernández Parrat, 2006; Mercado, 2012) y mejora del medioambiente así como de un equilibrio entre desarrollo y conservacionismo. Frente a las funciones, se reflexionaba sobre las disfunciones: catastrofismo y sensacionalismo, manipulación ideológica o comercial, superespecialización (lenguaje excesivamente técnico) y esteticismo, lo que algunos denominan en la jerga profesional televisiva "postalear", es decir, caer en la literatura o las imágenes bellas sobre la Naturaleza sin ofrecer ninguna información noticiosa. El periodista José $\mathrm{M}^{\mathrm{a}}$ Montero $(1999,2005,2008,2013)$ insiste en la clara tendencia a la superficialidad cuando se trata de informar sobre medio ambiente, prestando una atención desmesurada a elementos subalternos de la información, con grave descuido de los elementos principales. "Esta intrascendencia se manifiesta en ignorar las conexiones y efectos que determinados problemas ambientales tienen sobre el medio humano, quedando reducidos a conflictos más o menos coyunturales y, a veces, hasta anecdóticos" (2013: 148).

El repaso por las fuentes disponibles en la especialización tenía una aplicación inmediata en el proyecto de reportaje televisivo que los alumnos, en grupo, debían realizar como trabajo de curso. En el tema 3, los estudiantes conocían las principales publicaciones, se escuchaban o visionaban programas y se estudiaban los informes sobre la información ambiental en los medios, sobre todo los referidos a la información en televisión realizados por Bienvenido León (2007) y Pablo Francescutti (2009). Los últimos temas se dedicaban a analizar formatos (por ejemplo documentales) o casos concretos como el de Doñana (Elías, 2001) o el Prestige (Vicente Mariño, 2010).

En el curso 2010-11 se decide cambiar el temario eliminando como tales los temas 4 y 5. Los contenidos básicos que se impartían en ellos pasan al tema 1; los del tema 4 al punto 1.1 y los del tema 5 al 1.3. Como novedad, aparece como tema 4 el dedicado a Informar sobre Cambio Climático (CC). Tras varios años impartiendo la asignatura, consideramos grave la carencia de conocimientos básicos al respecto de los alumnos de Quinto Curso en un tema que ha alcanzado gran relevancia en la agenda internacional tras el documental de Al Gore 'Una verdad Incómoda', la concesión del premio Nobel de la Paz a Barak Obama y el Panel Intergubernamental de Expertos 
sobre el Cambio Climático (IPCC) y las expectativas creadas en la Cumbre del Clima celebrada en Copenhague en 2009.

Este tema incluye cinco epígrafes: conceptos básicos sobre el CC; proceso internacional de lucha contra el CC; fuentes de información sobre el CC; la sociedad ante el CC: conocimientos, creencias y comportamientos; recomendaciones para informar sobre el CC. Estos dos últimos puntos repasan las destacadas aportaciones de Pablo A. Meira $(2009,2010)$ que se convierte en materia de examen.

La inclusión de un tema específico sobre CC es muy satisfactoria en cuanto a resultados de conocimiento y en los cursos siguientes se opta por incidir en este tipo de contenidos centrados en la explicación, debate en clase y realización de prácticas de redacción y producción informativa sobre temas específicos. Estos son los destacados en el libro Lo Lógico de lo ecológico editado por APIA (2010), que se reparte en clase a todos los alumnos. El temario queda como sigue:

Tema 1. El Periodismo Ambiental como área de especialización.

1.1. Origen y evolución de la especialidad.

1.2. Funciones y disfunciones.

1.3 Fuentes en el periodismo ambiental.

Tema 2. Los temas de la información ambiental I.

2.1 Agua 2.2 Biodiversidad 2.3 Residuos 2.4 Energía 2.5 Salud 2.6 Otros

Tema 3. Los temas de la información ambiental II. Cambio Climático (CC)

3.1 Conceptos básicos sobre el CC.

3.2 El proceso internacional de lucha contra el CC.

3.3 La sociedad ante el CC: conocimientos, creencias y comportamientos.

3.4 Recomendaciones para informar sobre el CC

Tema 4. Formatos y medios para la información sobre medioambiente (con mismos epígrafes que el anterior).

La energía, asunto tratado por Pepa Mosquera en el libro de APIA, es uno de los temas en los que más se inciden, como objeto de estudio del proyecto $\mathrm{I}+\mathrm{D}+\mathrm{i}$ con financiación estatal "Análisis del tratamiento informativo de las políticas energéticas en España, procesos de recepción y organizaciones sociales" (Referencia CSO201238363).

\subsection{Los temas del periodismo ambiental: la cuestión energética}

Desde la práctica profesional, Joaquín Fernández (1995), el que fuera el creador del especio 'Reserva natural' en RNE y primer presidente de la Asociación de Periodistas Ambientales (APIA), diferenciaba dentro de la especialidad ambiental otras especialidades según su temática. Junto al que denominaba 'periodismo conserva- 
cionista', que atiende a preservación de la naturaleza en línea con la definición de Esteve y Fernández del Moral, el periodista señala el periodismo ambientalista, en el que entrarían aquellos asuntos vinculados a la contaminación (de las ciudades, del agua, acústica, lumínica, residuos) y distingue entre periodismo tecnocientífico, energético y ecopolítico (1995). El periodismo energético trataría sobre la energía, el modelo energético vigente y quienes lo promueven: petróleo, eléctricas, problemas derivados del uso de la energía como el calentamiento del planeta, el uso de energías alternativas. Aquí aparecen asuntos complejos, técnicos.

La cuestión energética merece ser considerada como una subespecialidad dentro del periodismo ambiental y es de hecho uno de los asuntos más relevantes en la agenda internacional. Según el informe World Energy Outlook (International Energy Agency, 2012), el mundo está fracasando en conducir el sistema energético mundial por una senda más sostenible. La demanda mundial de energía crece más de un tercio en el periodo que va hasta 2035; China, la India y Oriente Medio representan el 60\% de dicho aumento. Pese al crecimiento de las fuentes de energía de baja emisión de carbono, los combustibles fósiles siguen siendo predominantes en el mix energético mundial. WWF en su "Informe Planeta Vivo 2012", una evaluación bianual que analiza la situación de la biodiversidad global y mide la demanda de la población sobre los recursos naturales de la Tierra, destaca que la demanda de recursos de la humanidad excede en cerca de un 50\% la capacidad regeneradora del planeta, por lo que estamos destruyendo el capital natural en lugar de utilizar tan sólo los intereses, que sería lo sostenible. "Si seguimos con este ritmo, en 2030 necesitaremos 2 planetas para satisfacer nuestra demanda de recursos naturales". La organización insiste en que es imprescindible aunar los esfuerzos para cambiar el modelo energético actual (WWF, 2012).

Sin embargo, los ciudadanos no parecen estar muy informados sobre estos asuntos. Pérez-Díaz y Rodríguez (2008: 49) comprobaron en una encuesta a población joven (1.203 individuos de entre 16 y 35 años llevada a cabo en 2004 cómo los niveles de información sobre energía y medio ambiente dejaban mucho que desear. Sobre los usos y consumos de la energía, sus fines y procedencias, su nivel de información parece modesto, pero es todavía más bajo en lo relativo a los costes de la energía. El 38\% ni siquiera respondió a la pregunta sobre la fuente de la que procedía la electricidad de uso doméstico. En 2007, esta vez con una encuesta a personas de entre 18 y 75 años (1200 entrevistas), una vez más, "la impresión que transmiten los resultados es de conocimiento bajo", con algunos matices. Según el Centro de Investigaciones Sociológicas (CIS, 2007), un 59\% de la población se reconoce poco o nada informado. Por todo ello, consideremos de máximo prioridad investigar la cuestión energética en los medios y tratar el asunto en las aulas de Periodismo. 


\section{La información sobre medio ambiente en los Fundamentos del Periodismo}

La implantación del Espacio Europeo de Educación Superior ha provocado tres tipos de profundas transformaciones en las universidades: estructurales, curriculares y organizativas. Las primeras han modificado las etapas educativas y han supuesto en la práctica la eliminación del quinto curso de la antigua licenciatura de Periodismo. "Las curriculares han afectado a la definición, diseño y desarrollo del currículum y las organizativas a las condiciones en las que se desarrolla el proceso de enseñanza/ aprendizaje" (De Haro, 2012:15).

En el marco de estas nuevas condiciones que han supuesto la eliminación de la asignatura Periodismo Ambiental del Plan de Estudios del Grado de Periodismo, se han desarrollado diversas experiencias docentes-investigadoras con el objetivo de integrar la actualidad y especialización ambiental en las materias que conforman el que, según el primer Congreso Mundial sobre la Enseñanza del Periodismo (UNESCO, 2007), ha de configurar un módulo inicial en todo proceso formativo en Periodismo, denominado Fundamentos del Periodismo. En este módulo se proponen materias como 'Lógica pruebas e investigación', 'Redacción', 'Instituciones nacionales e internacionales' y 'Conocimientos generales'. Todas ellas, tal y como lo conciben los expertos, se impartirían en el primer año y pretenden sentar las bases para comprender la importancias de la profesión (Pereira et al, 2012: 139).

Para la integración de la especialización ambiental en materias básicas, en nuestro caso Fundamentos del Periodismo, optamos por ceñirnos a la cuestión energética como asunto de actualidad permanente e interés investigador, como hemos explicado anteriormente. La primera investigación-acción educativa (García-Valcárcel, 2009; Gil pascual, 2004; Latorre, 2003; Zabalza, 2003) este sentido fue llevada a cabo en 2011 tras la catástrofe de Fukushima (Mercado y Sánchez, 2011). El estudio que se presenta en este artículo insiste en la problemática nuclear pero centrándose en la construcción del Almacén Temporal Centralizado de residuos radioactivos en Villar de Cañas, Cuenca.

El Consejo de Ministros de 30 de diciembre de 2011 daba luz verde a la propuesta del ministro de Industria, Energía y Turismo, José Manuel Soria, para que el ATC de residuos nucleares se instalara en la localidad de Villar de Cañas (Cuenca). Actualmente, sólo existe el centro de El Cabril (Córdoba), donde se tratan residuos de baja y media actividad, por lo que el resto de residuos son almacenados en piscinas de las propias centrales nucleares hasta que son desmanteladas. La primera fase de construcción del ATC supondría una inversión de 384 millones de euros y la creación de 500 puestos de trabajo hasta 2015. En las fases siguientes, se invertirían 316 millones más y se crearían otros 150 puestos de trabajo, según las autoridades.

En esta investigación, el objetivo es integrar el debate energético en el aula a partir de la puesta en marcha de un experimento basado en las investigaciones realizadas sobre el impacto cognitivo de los encuadres noticiosos (framing effect). 


\subsection{Metodología}

Diversos estudios llevados a cabo sobre el procesamiento y los efectos de las noticias concluyen que la manera en que se enfoca un tema influye en la percepción que el público desarrolla sobre ese asunto, es decir, el tipo de encuadre utilizado para confeccionar una información ejerce un impacto significativo y congruente en las respuestas cognitivas de los sujetos (Scheufele, 1999, 2000; Valkenburg, Semetko y de Vreese, 1999; de Vreese, 2003, 2004; de Vreese y Boomgaarden, 2003) o en las actitudes, creencias y en el nivel de complejidad cognitiva con que las personas reflexionan sobre los asuntos sociales (Iyengar, 1991).

Los efectos de los encuadres noticiosos en la información política han sido los más investigados. En España, el equipo dirigido por Juan José Igartua en la Universidad de Salamanca ha analizado los efectos de los encuadres en la información sobre inmigración en relación a si los medios incitan o no a la xenofobia (Igartua y Muñiz, 2004a; Igartua, Muñiz, Otero, Cheng y Gómez, 2008; Igartua y Cheng, 2009).

En términos generales, las investigaciones experimentales sobre el efecto señalan que la manera en que se enfoca un tema (variable independiente) influye en la percepción (variable dependiente) que el público desarrolla sobre ese asunto (Igartua y Humanes, 2004). En esta investigación, la variable dependiente es el factor observado y medido para determinar el efecto de la variable independiente. Siguiendo por tanto estas investigaciones, nos centramos en el análisis de los efectos de los encuadres noticiosos en la información sobre la construcción del Almacén Temporal Centralizado (ATC) en Cuenca para promover después un debate en el aula y una práctica en la que los estudiantes planteen un proyecto de reportaje sobre el asunto.

Para conocer qué piensan los estudiantes sobre la cuestión energética en general y sobre la construcción del ATC en particular, se opta por la encuesta (Rojas, 1998) como herramienta para la recogida de datos. La muestra no probabilística estuvo formada por 210 estudiantes de Ciencias de la Información, de entre 18 y 32 años: 70 de la Universidad CEU Cardenal Herrera, 70 de la Universitat de València y 70 de la Universidad de Castilla la Mancha. La recogida de los datos se realizó las semanas del 22 y el 29 de abril de 2013, de manera colectiva en el contexto del aula de las tres universidades, con participación voluntaria. Los estudiantes cumplimentaron un cuestionario ad hoc.

Una vez recogida la información acerca de las creencias del alumnado acerca de la conveniencia del uso de la energía nuclear, en un lapso de tiempo relativamente breve (entre 2 y 4 horas), los alumnos son expuestos al visionado de dos noticias emitidas en Televisión Española sobre la construcción del ATC con encuadres noticiosos distintos previamente seleccionados por los profesores investigadores (Noticia1: La construcción del ATC creará unos 500 empleos, emitida el 09 de noviembre de 2012; Noticia 2: Cientos de personas protestan en Villar de Cañas por la instalación del ATC, emitida el 12 de febrero de 2012). En cada Universidad, la mitad de los alumnos visiona la noticia A y la otra mitad la noticia B. 
La mayoría de las investigaciones sobre el efecto framing en Comunicación ha utilizado como opción metodológica la investigación experimental que permite la manipulación sistemática de los encuadres noticiosos y, a su vez, la observación del impacto en condiciones controladas. Igartua, Otero, Muñiz, Cheng y Gómez (2006) en su estudio sobre los efectos cognitivos y afectivos de los encuadres noticiosos de la inmigración, construyeron las noticias con encuadres noticiosos determinados a partir de sus propias investigaciones previas de análisis de contenido (Igartua, Muñiz, Cheng, 2005; Igartua, Muñiz, Calvo, Otero y Merchán, 2005) y de noticias publicadas en la época en la que se desarrolló el estudio. En nuestro caso, los materiales son noticias reales emitidas por Televisión Española.

El objetivo era determinar si el visionado de la noticia provocaba cambios en la percepción del uso de la energía nuclear, es decir, evidenciar empíricamente los efectos de los encuadres noticiosos en los informativos televisivos. Así, en este contexto experimental, ante la pregunta: ¿si hubiera tenido que participar en un referéndum acerca de la conveniencia o no de la instalación del ATC en su localidad de residencia, qué hubiera votado?, antes (PRE) y después (POST) de visionar la noticia propuesta, la hipótesis es que los posibles cambios de percepción dependerán del encuadre noticioso de las informaciones a las que han sido expuestos los sujetos.

Bajo los presupuestos de la estadística inferencial para la resolución cuantitativa de la investigación, se creó un libro de códigos con las variables consideradas y se procedió al análisis correlacional multivariable junto a los estadísticos de contraste x2 (Ji- cuadrado), y r ( $\mathrm{r}-\mathrm{y})$ de Pearson con los que resolver la hipótesis de partida y conocer el grado o fuerza de asociación entre las variables propuestas. Para evaluar la consistencia interna de la investigación se ha empleado el coeficiente $(\alpha)$ de Cronbach. Con el software estadístico SPSS v.17.0, se realizó una tabla de contingencia o Crosstabulation.

\subsection{Resultados}

La mayoría de los 210 alumnos encuestados están en contra de la energía nuclear y hubiera no votado NO a la posible construcción del ATC en su localidad (67,2 por ciento). La mitad de los estudiantes (en las tres universidades) considera que la energía nuclear debería desaparecer como fuente de energía en España.

La prueba $t$ para muestras relacionadas muestra que únicamente puede ser considerado como tendente la opinión modificada del grupo de la UCM (500 empleados) en claro acuerdo con la instalación de la ATC como creadora de empleos directos.

Tras el visionado de las noticias, se procedió al debate sobre el asunto y una vez analizados los datos, se instó a los alumnos a que buscaran información, puesto que cerca del veinte por ciento indican estar poco informados respecto a un asunto de tal importancia por lo que votarían en blanco ( $\mathrm{n}=39$, un 18,5 por ciento).

La realización del estudio sobre el efecto de los encuadres noticioso, el debate en clase y la búsqueda de información para la preparación de un proyecto de reportaje en 
profundidad ha supuesto una experiencia muy satisfactoria tanto para los profesores investigadores como para los alumnos que participaron.

\section{Conclusiones}

El periodismo ambiental como especialización periodística ha ido adquiriendo mayor importancia en las últimas décadas al incrementarse el conocimiento y la preocupación sobre las consecuencias de nuestro modo de vida en el planeta. Los asuntos ambientales no se asocian ya únicamente a los relacionados con la pérdida de la biodiversidad (por ejemplo, animales en peligros de extinción) sino que se perciben como una realidad imbricadas en la realidad política y económica. En este sentido, la cuestión energética aparece en la mayor parte de los grandes problemas ambientales: cambio climático, deforestación, contaminación...

La asignatura Periodismo Ambiental permitía trabajar estos temas durante un cuatrimestre, analizar noticias en distintos soportes y producir información periodística especializada. Su eliminación de los planes de estudio obliga a reflexionar en torno a la manera en la que introducir esta realidad en los contenidos de asignaturas como Fundamentos de la Comunicación Periodística, Periodismo en radio y televisión o Periodismo Especializado, impartidas en la Universidad CEU Cardenal Herrera).

El experimento realizado en esta Universidad, en la Universidad de Valencia y en la Universidad de Castilla-La Mancha ha confirmado que la capacidad de comprensión y análisis de proposiciones complejas como el paradigma energético mundial puede incrementarse fundamentando y poniendo en marcha en el aula métodos de trabajo que potencien el aprendizaje significativo de los estudiantes de Comunicación.

El periodismo ambiental no supone una teorización sobre ecología sino la reflexión crítica acerca de cómo informar sobre problemas como la escasez energética, es decir, la incorporación de esta temática en los procesos de selección, interpretación y narración de la actualidad así como en los modos de actuación profesional que se corresponden con ellos. Los Grados en Periodismo no deben dejar de lado este ámbito de la actualidad.

\section{Bibliografía}

APIA (2010). Lo lógico de lo ecológico. Madrid: Fundación Biodiversidad. CERRILlO, A. (Coord.).(2008). El periodismo ambiental. Análisis de un cambio cultural en España. Madrid: Fundación Gas Natural. 
DE HARO, V. (Coord.) (2012). Novedades docentes en el EEES. Madrid: Visión Libros.

DE VREESE, C. H. (2003). Framing Europe: Television news and European integration. Amsterdam: Aksant Academic Publishers.

DE VREESE, C. H. (2004). "The effects of frames in political television news on issue interpretation and frame saliente". En: Journalism and Mass Communication Quarterly, $\mathrm{n}^{\circ}$ 81, p. 36-52.

DE VREESE, C.H. \& BOOMGAARDEN, H. (2003). "Valenced news frames and public support for the UE". En: Communications, n" 28 (4), p. 36-381.

ELIAS, C. (2001): "Periodismo especializado en medio ambiente: el caso Doñana como paradigma de manipulación informativa". En: Ámbitos. Revista internacional de comunicación, $\mathrm{n}^{\circ} 6$.

ENTMAN, R. (1993). "Framing: toward clarification of a fractured paradigm". En: Journal of Communication, $\mathrm{n}^{\circ} 43$ (4), p. 51-58.

ESTEVE, F. y FERNÁNDEZ DEL MORAL, J. (1999). Áreas de Información Periodistica Especializada. Madrid: Fragua.

FERNÁNDEZ PARRATT, S. (2006). Medios de comunicación y medio ambiente. Madrid: Fragua.

FERNÁNDEZ REYES, R. (2003). "En torno al debate sobre la definición del periodismo ambiental". En: Ámbitos , nº 9-10.

FERNÁNDEZ REYES, R. (2004). "Periodismo ambiental y periodismo sostenible". En: Ámbitos, n'. 11-12.

FERNÁNDEZ REYES, R. (2011). “Aproximación a la bibliografía del periodismo ambiental iberoamericano". En: Razón y Palabra, no 77. http://www.razonypalabra.org.mx/varia/77\%202a\%20parte/12_Fernandez_V77.pdf [Consultado el 23-11-2012]

FERNÁNDEZ SÁNCHEZ, J. (1995). Periodismo ambiental en España. Madrid: Ministerio de Obras Públicas, Transportes y Medio Ambiente.

FRANCESCUTTI, P., et al (2009). El cambio climático y la crisis ecológica en los informativos. Un análisis de la información ambiental televisiva. Madrid: APIA Universidad Rey Juan Carlos.

GARCÍA-VALCÁRCEL, A. (coord.) (2009). Experiencias de innovación docente universitaria. Salamanca: Universidad de Salamanca.

GIL PASCUAL, J. A. (2004): Bases metodológicas de la investigación educativa: análisis de datos. Madrid: UNED.

INTERNATIONAL ENERGY AGENCY (2012). Resumen Ejecutivo World Energy Outlook. En: http://www.iea.org/publications/freepublications/publication/Spanish.pdf [Consultado el 28-10-2013].

IGARTUA, J. J. (2006): Métodos cuantitativos de investigación en comunicación. Bosch. Barcelona.

IGARTUA, J. J. \& MUÑIZ, C. (2004a). "Encuadres noticiosos e inmigración. Un análisis de contenido de la prensa y televisión españolas". En: Zer. Revista de Estudios de Comunicación, $\mathrm{n}^{\circ}$ 16, p. 87-104. 
IGARTUA, J. J. \& CHENG, L. (2009): "Effect of Group Cue While Processing News on Immigration". En: Journal of Communication, $n^{\circ}$ 59, p. 726-749.

IGARTUA, J. J. \& HUMANES, M. L. (2004b): Teoría e investigación en comunicación social. Madrid: Síntesis.

IGARTUA, J. J.; MUÑIZ, C. ; OTERO, J.; CHENG, L. \& GÓMEZ, J. (2006b). "Efectos cognitivos y afectivos de los encuadres noticiosos de la inmigración". En V Jornadas Medios de comunicación, inmigración y sociedad. Universidad de Salamanca.

IGARTUA, J. J. et al (2008): "Recepción e impacto socio-cognitivo de las noticias sobre inmigración”. En: Revista de Psicología Social, no 23 (1), p. 3-16.

IYENGAR, S. (1991). Is any responsable? How television frames political issues. Chicago: The University of Chicago Press Book.

LATORRE BELTRÁN, Antonio et al. (2003): Bases metodológicas de la investigación educativa. Barcelona: Experiencia.

LEÓN, B. (2007). "El medio ambiente en las televisiones españolas". En Cultura verde: ecología, cultura y comunicación. Sevilla: Consejería de Medio Ambiente, Junta de Andalucía.

MEIRA, P. A. (2009). Comunicar el Cambio Climático. Escenario Social y líneas de acción. Madrid: Ministerio de Medio Ambiental. Organismo Autónomo de Parques Naturales.

MERCADO, M. (2012): "Periodismo Ambiental, entre la especialización y el compromiso". Prensa y Periodismo Especializado V. Guadalajara: Asociación de la Prensa de Guadalajara, p. 439-446.

MERCADO, M. y SÁNCHEZ, S. (2012): "La integración de la educación ambiental en la formación periodística como proyecto de innovación docente". En: Vivat Academia, no 117 , p. 576-597.

MONTERO, J. M. (2013). "El cambio global y la crisis del periodismo". En Medios de Comunicación y Cambio Climático. Sevilla: Fénix editora.

MONTERO, J. M. (2008): “Información Ambiental en televisión”. En El periodismo ambiental. Análisis de un cambio cultural en España. Barcelona: Fundación Gas Natural.

MONTERO, J.M. (2005). El medio en los medios. Ayuntamiento de Sevilla.

MONTERO, J.M. (1999): "El rol ambiental de los medios de comunicación”. En: Revista Ciclos $\mathrm{n}^{\mathrm{o}} 5$.

PEREIRA, X., et al (2012). "Adaptación de las propuestas de la Unesco sobre la formación de periodistas a la docencia universitaria. En Novedades docentes en el EEES. Madrid: Visión Libros.

PÉREZ-DÍAZ, V. y RODRÍGUEZ, J.C. (2008). Energía y Sociedad. Actitudes de los españoles ante los problemas de la energía y del medio ambiente. Madrid: Club Español de la Energía.

ROJAS TEJADA, A. J. et al. (1998): Investigar mediante encuestas. Fundamentos teóricos y aspectos prácticos. Síntesis: Madrid. 
SCHEUFELE, D. A. (2000). “Agenda-setting, priming, and framing revisited:Another look at cognitive effects of political Communications". En: Mass Communication \& Society, $\mathrm{n}^{\circ}$ 3, p. 297-316.

SCHEUFELE, D. A. (1999): "Framing as a theory of media effects". En: Journal of Communication, $n^{\circ} 49$, p. 103-122.

VALKENBURG, P. M., SEMETKO, H. A. y DE VREESE, C.H. (1999): “The effects of news frames on readers' thoughts and recall". En: Communication Research, $26(5)$, p. 550-569.

VARILLAS, B. (2007). "Historia de la información ambiental en España". En curso Comunicación y medio ambiente: el poder creciente de la información medioambiental. Segovia: CENEAM. Organismo Autónomo Parques Nacionales-Ministerio de Medio Ambiente.

VICENTE MARIÑO, M. (2010). "Agendas, encuadres y discursos en los noticiarios televisivos españoles durante la crisis del Prestige". En: Athenea Digital. Revista de Pensamiento e Investigación Social, $\mathrm{n}^{\circ}$ 19, p. 249-257.

WWF (2012). Informe Planeta Vivo. En:http://www.wwf.es/noticias/informes_y_ publicaciones/informe_planeta_vivo_2012/[Consultado el 28-10-2013]

ZABALZA, M. A. (2003): Competencias docentes del profesorado universitario. Madrid: Narcea.

\section{Los autores}

María Teresa MERCADO SÁEZ. Doctora en Ciencias de la Información, UCM (2005), Licenciada en Periodismo UPV (1996), Licenciada en Humanidades, UV (2007) y Máster en Gestión Cultural, CEU (2005). Profesora de Periodismo en la Universidad CEU Cardenal Herrera (Valencia, España). Directora del Instituto CEU de Investigación de Disciplinas Económicas, Ambientales y Sociales (IDEAS). Investigadora Principal del Grupo Sostenibilidad y Periodismo Especializado y miembro del Grupo de Investigación en Discapacidad y Comunicación (GIDYC) de la UCH, ha publicado en revistas como Journalism Studies, Estudios sobre el Mensaje Periodístico, Razón y Palabra o la Revista ZER. Es autora de varios capítulos de libros sobre Periodismo Especializado.

Sebastián SÁNCHEZ CASTILLO. Doctor en Ciencias de la Comunicación, licenciado en Comunicación Audiovisual y en Historia del Arte. Es profesor del departamento de Teoría de los Lenguajes y Ciencias de la Comunicación de la Universidad de Valencia. Es miembro de los grupos de investigación Sostenibilidad y Periodismo Especializado y Discapacidad y Comunicación (GIDYC) de la Universidad CEU UCH. Entre sus publicaciones recientes destacan "Narrativa audiovisual y discapacidad. Realización televisiva comparada de los Juegos Olímpicos y Paralímpicos de Pekín 2008” en la Revista ZER (2011) y "El análisis crítico de la cuestión energética: internacionalización y competencias profesionales en Periodismo", en Estudios sobre el Mensaje Periodístico (2013). 
José María HERRANZ DE LA CASA. Doctor en Periodismo por la Universidad Complutense de Madrid. Ha trabajado como periodista en el diario MARCA y ha sido profesor de la Universidad Católica de Ávila y de la Universidad Europea Miguel de Cervantes. En ambas universidades fue también director del Gabinete de Comunicación. En la actualidad es profesor de Periodismo en la Universidad de Castilla-La Mancha. Tiene distintos trabajos publicados en torno a las líneas de investigación que desarrolla: la comunicación y transparencia en las ONG; la comunicación empresarial y organizacional; y la responsabilidad social. Forma parte de unproyecto de investigación, financiado por el gobierno español, que analiza el tratamiento informativo de las políticas energéticas en España. 